\title{
Erratum: Pumping current in a non-Markovian $N$-state model [Phys. Rev. Research 3, 023238 (2021)]
}

Ville M. M. Paasonen (ㄷ) and Hisao Hayakawa

(Received 20 July 2021; published 28 July 2021)

DOI: 10.1103/PhysRevResearch.3.039002

We have realized that there are some minor errors in this paper, which we correct here. The results and conclusions of our paper are not changed by these corrections.

First, we clarify our discussion about the condition of detailed balance, starting above Eq. (4) of the paper. The condition discussed there is that of existence of an equilibrium state for which it is sufficient to have det $L=0$. However, detailed balance is a stricter condition and does not necessarily hold even when the determinant of the transition matrix is vanishing. For instance, an equilibrium state could contain cyclic hopping chains, which would violate detailed balance.

Equations (18) and (20) describing the calculation of the cumulant generating function using full counting statistics have some typographical errors. In Eq. (18), the integral is not needed, and the correct expression reads

$$
\overline{z(\chi)}=z(2 \pi, \chi)
$$

This is because the solution of Eq. (17) already contains the integral with respect to the phase $\theta$. Equation (20) lacks a prefactor arising from the unit system employed in this paper. The correct expression reads

$$
g(\chi)=\frac{\Omega}{2 \pi} \ln \overline{z(\chi)}
$$

The remaining two corrections pertain to Appendix B of our this paper. There are some typographical errors in Eq. (B14), which shows a perturbative expansion of $\delta_{i}$. This equation should read

$$
\delta_{i}=\delta_{i}^{(0)}+\sum_{n=1}^{\infty}\left(\frac{\Omega}{\lambda_{i}} \frac{d}{d \theta}\right)^{n} \delta_{i}^{(0)} .
$$

Finally, we noted that some of the plots in Figs. 12 and 13 of this paper are not correctly labeled. Specifically, the fifth and the sixth components of the right eigenvectors in both of these figures are reversed. The correct labelings are shown in Figs. 12 and 13 of this Erratum. We can now confirm a symmetry property of the eigenspectrum. Since the transition matrix is invariant under the

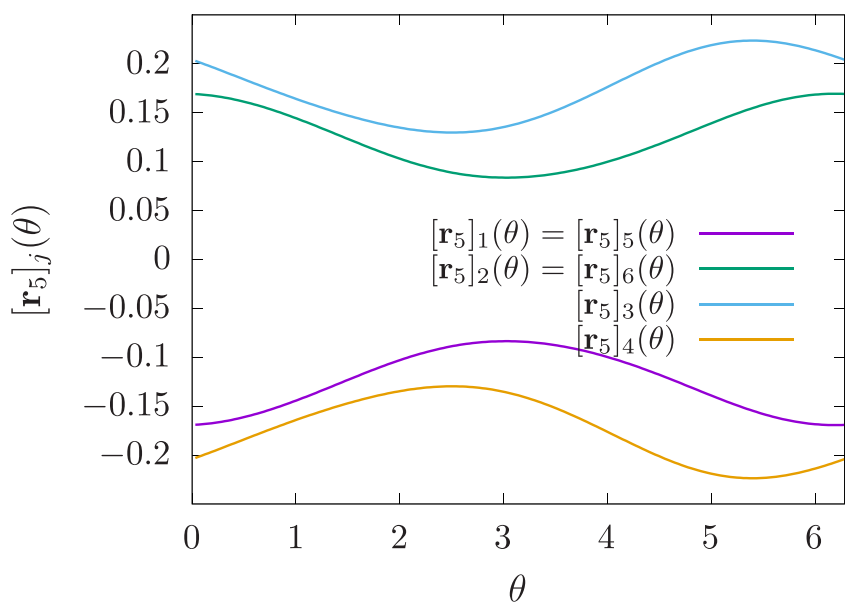

FIG. 12. The six components of the eigenvector $\boldsymbol{r}_{5}$ of the $N=2$ system plotted against $\theta$ with $r=0.9, \phi=\pi / 2$, and $w=2$. 


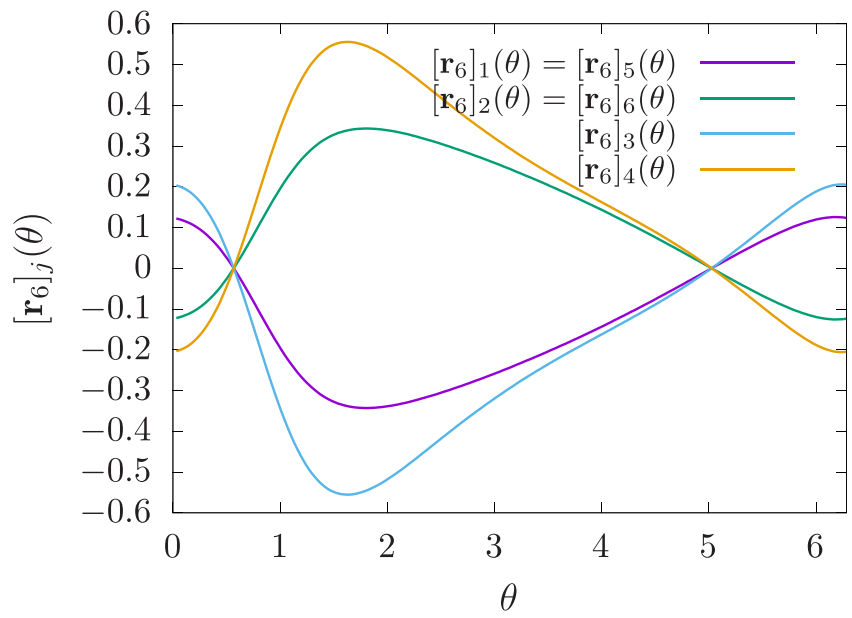

FIG. 13. The six components of the eigenvector $\boldsymbol{r}_{6}$ of the $N=2$ system plotted against $\theta$ with $r=0.9, \phi=\pi / 2$, and $w=2$.

transformation $\left[\rho_{1}, \rho_{2}, \rho_{3}, \rho_{4}, \rho_{5}, \rho_{6}\right]^{T} \rightarrow\left[\rho_{6}, \rho_{5}, \rho_{4}, \rho_{3}, \rho_{2}, \rho_{1}\right]^{T}$, all eigenvectors should be either symmetric or antisymmetric with respect to this operation. We can see from the plots in this paper that $\boldsymbol{r}_{1}, \boldsymbol{r}_{2}$, and $\boldsymbol{r}_{4}$ are symmetric and $\boldsymbol{r}_{3}$ antisymmetric with respect to this transformation. We can further confirm from the corrected plots of Figs. 12 and 13 that $\boldsymbol{r}_{5}$ and $\boldsymbol{r}_{6}$ are antisymmetric.

We thank S. Takesue for his indication of our errors. 